\title{
Performance of an Optical Stabilization System at NSLS Beamline U12IR \\ R.J. Smith ${ }^{1}$, E. Stavitski ${ }^{1}$, and G.L. Carr ${ }^{1}$ \\ ${ }^{l}$ Photon Sciences, Brookhaven National Laboratory, Upton, 11973, NY, USA
}

\begin{abstract}
A low-cost optical feedback system using dynamic mirrors has been developed at the NSLS for stabilizing the position and direction of an infrared synchrotron beam against thermal drift and mechanical noise. The system design has some unique features that potentially simplify installation into an existing infrared beamline. We describe the system and its features along with some performance results.
\end{abstract}

\section{Keywords}

beam stabilization, dynamic mirror, feedback, noise reduction, infrared synchrotron beamline

\section{Introduction}

At many synchrotron light source facilities with operating infrared beamlines, the signal detected in the infrared spectrometer is less than ideal due to the relative movement of the optical components and the electron beam in the accelerator itself. This can be especially problematic for facilities that are not operating in "top up" mode. As a result, the large variation in beam current and the associated heating of mechanical components can vary by factors of 3 over a period of hours. The result is an effective source whose position appears to shift in time (from thermal expansion) and also "wobbles" due to additional motion from mechanical vibrations and electrical systems. Both of these introduce noise and error that limit the spectroscopic performance for experimenters using the facility

This problem was first tackled successfully at the Advanced Light Source[1] (ALS, Lawrence Berkeley National Laboratory) using a mixture of commercial components and custom-designed \& built electronics. This was followed by similar installations at other synchrotron light source facilities operating infrared beamlines[2,3]. The subsequent expansion of commercial products (developed for stabilizing laser beams) has opened the possibility for constructing a lower cost system from almost entirely off-the-shelf components. Here we describe such a system and demonstrate its performance at the National Synchrotron Light Source VUV/IR ring at Brookhaven National Laboratory. This particular electron storage ring was not originally designed as an ultra-stable light source so the magnitude of apparent beam motion is measured in hundreds of micrometers and angular deviations measured in hundreds of microradians, probably among the largest to be encountered at a synchrotron radiation facility. Additionally, we consider a system layout that differs from those found elsewhere and may allow for additional flexibility in deployment at a typical synchrotron infrared beamline.

*Corresponding author. Tel.: +16313448033; Fax: +16313447039;

E-mail address: rsmith@bnl.gov (R.J. Smith). 


\section{Stabilization System Design}

The basic design of an optical beam stabilization system consists of a pair of electrically controlled X-Y tilt mirrors (TMs) that serve to steer the beam in two orthogonal planes (e.g. vertically and horizontally). These are typically flexure-type optical mounts with piezoelectric actuators to provide a reasonably rapid response. The beam reflected from these mirrors is sampled using a pair of position sensitive detectors (PSDs) that can measure the lateral displacement of the light beam from its central reference position (again in both horizontal and vertical directions transverse to the beam direction). When the beam is not centered on the PSD, a deviation signal is produced that is proportional to the lateral displacement. This signal is then used to drive the tilt mirrors until the deviation is zero, usually in combination with a PID (proportional/integral/derivative) control system that is common to most feedback systems for improved performance and stability. The result is a beam that is effectively "locked" to the PSDs. Assuming the PSDs are fixed to the infrared measurement system, the effect of beam motion is mostly eliminated. Of course there is a finite response time to the electrical and mechanical components of the stabilization system, thus the beam movement and accompanying noise are not instantly corrected - becoming progressively worse toward higher frequencies. Fortunately, most of the noise stems from mechanical systems driven by electrical motors and is primarily below $200 \mathrm{~Hz}$. The PSDs themselves typically use silicon detectors that sense wavelengths shorter than $1 \mu \mathrm{m}$, a spectral range included in most infrared beamline extractions, though not typically used for experiments. Therefore, a visible/infrared dichroic beamsplitter is a convenient way to provide light to the PSDs while directing infrared to the beamline instruments. We used a silicon window oriented near Brewster's angle to transmit infrared and reflect sufficient visible light to the PSDs. Other facilities have used commercial dichroic beamsplitters to reflect the infrared light and transmit the visible light to the relevant PSD.

The standard geometrical arrangement of the stabilization system's components has each tilt mirror followed by a dichroic beamsplitter and PSD, i.e. in sequence, with the deviation signal from the $1^{\text {st }}$ PSD driving the $1^{\text {st }}$ tilt mirror and the $2^{\text {nd }}$ PSD driving the $2^{\text {nd }}$ tilt mirror. A schematic layout is shown in Figure 1a and its geometric equivalent in Figure $1 b$. The geometry has the incident beam already on the proper axis to more clearly show the relationship between the angular positions of the two tilt mirrors and the lateral position deviations at the PSDs. In operation, the beam will be incident on a slightly different axis and the TMs will bring the beam to the proper axis (PSD deviations equal to zero). Though we described the apparent beam motion at NSLS as being large, it is still quite small relative to the dimensions of the geometrical layout. Still, one needs to place the components judiciously so that the overall range of the tilt mirrors is sufficient for correcting the beam position and to ensure the beam does not spill over the edge of a downstream optical element. This usually entails having TM1 near a source point where the lateral displacement from the ideal beam path is not large, and then having PSD1 and TM2 at a distance that puts the angular range of TM1 within the lateral position sensitivity and overall dimension of PSD1. In this configuration, a position deviation on PSD1 is corrected by a change in angle for TM1. But this, in turn, results in a deviation on PSD2 that is then corrected by TM2. Of course any initial deviation sensed at PSD2 is directly corrected without affecting PSD1. For such a system, the rate 
at which a deviation is corrected is determined primarily by the electronic and mechanical response of the tilt mirrors. Note that two dichroic beam splitters are needed for this geometry. Also, the need to have the two PSDs rigidly fixed to the infrared instrument table effectively forces all of the stabilization components (beamsplitters and tilt mirrors) to be dedicated to a particular infrared instrument. This can be inconvenient, or expensive, for a beamline that supports more than one endstation.

Figure 1a.

Figure 1b.

An alternative component arrangement, shown in Figure 2a, has both of the tilt mirrors placed before the two PSDs. Such an arrangement has the advantage of a single dichroic beamsplitter (reducing beam losses and system cost) and only the PSDs need to be fixed to the infrared instrument table. Since the PSDs are a small fraction of the total system cost, these could be economically replicated at each infrared instrument endstation while employing a single set of upstream tilt mirrors, drivers and feedback electronics. A disadvantage of this geometry is that the deviation corrections for PSD1 and PSD2 are more coupled. We will consider this issue in the next section.

Figure 2a.

Figure $2 b$.

\section{Performance Analysis}

We begin by noting some basic issues for a tilt mirror feedback system that can affect the system response time and ability to correct position deviations. One is the required dynamic range of the tilt mirror. Another is the transverse spatial sensitivity of the PSDs. These issues can be managed by varying the distance between a given tilt mirror and the relevant PSDs, by suitable choice of tilt mirror mechanics, and through the design and implementation of the piezoelectric actuators. There are also issues concerning the feedback controls connecting a given PSD output signal to the corrective drive signal, usually as part of a proportional-integral-derivative (PID) control loop. All of these will affect the response time for a given PSD/TM feedback loop and of the completed system. A full analysis of all these aspects is beyond the scope of this paper, so we limit ourselves to some specific layouts of a beam stabilization system appropriate for use in a synchrotron infrared beamline.

We can use the geometrical representations shown in Figures $1 b$ and $2 b$ to analyze some of the performance issues for various feedback schemes. Since we are only concerned with beam displacements from a reference direction, we consider the effect of the tilt mirrors as a direct angular deviation $\varphi$ of the incident beam, without including the overall change in direction due to reflection. Also, when the mirrors are used at near-normal incidence, the analysis for the two orthogonal directions for beam displacement $(x$ and $y$ ) are equivalent and decoupled, so we consider only one direction $(x)$. The angular range of the tilt mirrors is rather small, so we can immediately use the small angle approximation to yield linear relationships between the TM angles and PSD displacements. In general, the relation between the tilt mirror angles $\varphi_{1} ; \varphi_{2}$ and the displacements $x_{1} ; x_{2}$ sensed at the PSDs is of the form 


$$
x_{1}=A \varphi_{1}+B \varphi_{2} \text { and } x_{2}=C \varphi_{1}+D \varphi_{2}
$$

or, in matrix notation

$$
\left(\begin{array}{l}
x_{1} \\
x_{2}
\end{array}\right)=\left(\begin{array}{ll}
A & B \\
C & D
\end{array}\right)\left(\begin{array}{l}
\varphi_{1} \\
\varphi_{2}
\end{array}\right) \equiv \mathbf{R}\left(\begin{array}{l}
\varphi_{1} \\
\varphi_{2}
\end{array}\right)
$$

where $\mathbf{R}$ is the response matrix. In other words, for the general system, the beam position at a given PSD is a function of the angles for both tilt mirrors. To understand how the correction process functions for a coupled system, we must keep in mind that a basic system has the position deviation signal for a particular PSD driving the corrective response of one particular TM. Due to the finite response time of a given feedback loop, we can use an iterative approach to characterize the overall corrective response. In the setup of figure 1a, a position deviation $\delta x_{1}$ at PSD1 signals TM1 to produce a corrective angle change $\delta \varphi_{1}=-\delta x_{1} / A$. This results in a new deviation on PSD2, given as $\delta x_{2}=$ $C \cdot \delta \varphi_{1}=-(C / A) \cdot \delta x_{1}$. Such a deviation at PSD2 signals TM2 to adjust an amount $\delta \varphi_{2}=$ $-\delta x_{2} / D=(C / A D) \cdot \delta x_{1}$. The change in TM2 results in a deviation at PSD1 of $\delta x_{1}{ }^{\prime}=$ $B \cdot \delta \varphi_{2}=(B C / A D) \cdot \delta x_{1}$. Thus the result of one corrective feedback iteration of the system is a deviation reduced by a factor of $K \equiv \delta x_{1}{ }^{\prime} / \delta x_{1}=B C / A D$. A similar analysis for an initial deviation at PSD2, being corrected by a change in TM2, leads to the same ratio. Likewise, changing the feedback so that PSD2 controls TM1 and PSD1 controls TM2 yields the inverse, i.e. a reduction factor of $K \equiv A D / B C$. Obviously we desire $K$ to be as small as possible and certainly not greater than 1 , otherwise the deviation grows and the coupled system is unstable. Unless $B C=A D$, one of the two connection arrangements yields a converging system.

We can apply this analysis to the standard geometry (Fig. 1b) and assign variable $a$ for the distance from TM1 to PSD1, $b$ for PSD1 to TM2 and $c$ for TM2 to PSD2. The position deviation $x_{1}$ at PSD1 is related to an angular deviation $\varphi_{1}$ at TM1 as $x_{1}=a \cdot \varphi_{1}$. The deviation $x_{2}$ at PSD2 is related to the angular deviation from both TM1 and TM2, i.e. $x_{2}=(a+b+c) \cdot \varphi_{1}+c \cdot \varphi_{2}$. In our matrix notation we have $A=a, B=0, C=a+b+c$ and $D=c$. If we choose to have PSD1 supply the correction signal to TM1 and PSD2 to TM2, the relevant reduction ratio $K \equiv B C / A D=0$ and the system is not only stable but the deviation is fully corrected in one feedback iteration.

A similar analysis can be applied to the alternate arrangement of Figures $2 \mathrm{a}$ and $2 \mathrm{~b}$. Here we have the position deviation at each PSD related to the angular deviation for both tilt mirrors, i.e. $x_{1}=(a+b) \cdot \varphi_{1}+b \cdot \varphi_{2}$ and $x_{2}=(a+b+c) \cdot \varphi_{1}+(b+c) \cdot \varphi_{2}$. Thus we have $A=a+b$, $B=b, C=a+b+c$ and $D=b+c$. The correction reduction factor is now

$$
K \equiv \frac{B C}{A D}=\frac{b(a+b+c)}{(a+b)(b+c)}=\frac{a b+b^{2}+b c}{a b+b^{2}+b c+a c}
$$

which is clearly less than 1 but also not equal to zero. Another feedback cycle reduces the deviation another factor of $K$, etc. The overall result is an additional iterative factor in the feedback, i.e. slowing down the effective response time. Obviously, having the 
product $a c$ large and $b$ small is beneficial for achieving a more rapid convergence and faster response time.

In practice, we found that including a focusing lens for the visible light just downstream of the dichroic beam splitter was helpful in producing a properly sized spot on the PSDs. Since a lens functions by producing an angular deviation proportional to beam's distance from the lens center, its behavior can be introduced as a $3^{\text {rd }}$ tilt angle. As shown in figure 2c, we assign $\varphi_{3}=-d / f$ where $d$ is the lateral distance of the beam from the lens center and $f$ is the lens focal length.

Figure 2c.

The distance $d$ can be expressed in terms of the upstream tilt mirror angles and distances, in the same manner as the deviation at PSD1 in figure $2 b$ was calculated. For the geometry given in figure $2 \mathrm{c}$, we obtain:

$$
\begin{aligned}
& x_{1}=\left[f-\frac{c}{2}+\frac{(a+b) c}{2 f}\right] \varphi_{1}+\left[f-\frac{c}{2}+\frac{b c}{2 f}\right] \varphi_{2} \\
& x_{2}=\left[f+\frac{c}{2}-\frac{(a+b) c}{2 f}\right] \varphi_{1}+\left[f+\frac{c}{2}-\frac{b c}{2 f}\right] \varphi_{2}
\end{aligned}
$$

where $f$ is the lens focal length. Though the algebra is a bit more complex, the expressions are still of the form given by equations (1) and (2). Depending on the actual values for the various distances and the lens focal length, one can have $K>1$. Therefore, it can be necessary to have PSD1 control TM2 and PSD2 control TM1 to yield $K \equiv A D / B C<1$ for stability, consistent with experimental observations. For our specific geometry with $f=500 \mathrm{~mm}$ we obtained $K=0.6$, meaning it requires two iteration cycles for the deviation to be reduced to $1 / e$ of the original deviation value. It should be noted that the response matrix $\mathbf{R}$ for a constructed system can be directly measured by changing each tilt mirror a known amount and reading the deviation signal from each position sensitive detector. Note that our arrangement has the PSDs placed away from lens focus to ensure sensitivity to lateral beam displacements.

Lastly, we note that it should be possible to improve the feedback convergence by using a linear combination of the error signals for both PSDs to determine the corrective response of a given TM, thus eliminating this iterative effect. Note that our criteria of $B C \neq A D$ for a convergent solution to exist is equivalent to having the determinant of the matrix $\mathbf{R}$ be non-zero, which is a required condition for inverting the expressions relating the PSD error signal to the TM angle. We did not attempt to implement this in our demonstration system, but identify it as a future upgrade to improve the system's dynamic performance.

\section{System Details and Measured Performance}

Our beam stabilization system was developed for use at the U12IR beamline on the NSLS VUV/IR storage ring synchrotron light source at Brookhaven National Laboratory (Upton, NY, USA). This beamline is used mainly for microspectroscopy in the far and mid-IR spectral range. The endstation is a Spectra-Tech Ir $\mu s^{\mathrm{TM}}$ microspectrometer with a 
custom silicon beamsplitter to extend the available spectral range below the nominal cutoff of a $\mathrm{Ge} / \mathrm{KBr}$ beamsplitter. The moving mirror of the built-in rapid-scan spectroscopic interferometer produces optical velocities up to about $1 \mathrm{~cm} / \mathrm{s}$ such that mechanical noise in the $100 \mathrm{~Hz}$ range appears as intensity noise near a frequency of $100 \mathrm{~cm}^{-1}$, well within the nominally useful spectral range of the instrument when the synchrotron source is used. This makes far-infrared measurements particularly sensitive to beam noise. The beam stabilization system was assembled using mostly off-the-shelf components from several commercial vendors: Thorlabs (PSDs, PID controller, piezoelectric actuators and their drivers, various optical mounts and driver software), MDC Vacuum (electrical feedthroughs), Nor-Cal (ISO-80 vacuum cross and feedthroughs for mechanical beamsplitter adjusters), Siskiyou (flexure-type optical mounts), Edmund Optics (visible beamsplitter) and a personal computer running $\mathrm{LabView}^{\mathrm{TM}}$ for the control system. A diagram of the setup is shown in Figure 3.

Figure 3.

To include the stabilization system within the existing beamline optical system, three modifications were made: 1) the mirror mounts inside the vacuum enclosure (where the beam is collimated to match the microspectrometer's input aperture) were changed to flexure mounts with piezoelectric actuators, 2) the distance between the two flexure mounts was increased to have the quantity " $a$ " as large as possible and 3) a dichroic beamsplitter was placed in the beam just upstream of the microspectrometer. The dichroic beamsplitter was made from a thin piece of silicon oriented at 45 degrees to reflect a portion of the visible light out through a viewport while allowing the infrared to be mostly transmitted. The incident light was oriented for predominantly $p$-polarized reflection, allowing about $68 \%$ of the infrared to be transmitted and about $31 \%$ of the visible reflected. This gave sufficient visible light intensity for the PSDs to function optimally.

We operated the beam stabilization system over multiple beam injection and decay cycles. The time between injections during daytime operations is about 4-1/2 hours. Figure 4a shows the measured deviations for both X and Y positions on both of the PSDs (labeled "upstream" for PSD1 and "downstream" for PSD2) over the time period that the beam decays from $1000 \mathrm{~mA}$ down to $330 \mathrm{~mA}$. Approximately every 15 minutes we would turn the feedback electronics ON or OFF to see how well the system would recover the proper beam position. As can be seen, deviations of 100s of microns are quickly (fraction of a second) corrected and maintained below the 1 micron level. Note that the uncorrected Y-direction deviations passed through zero to negative values just prior to time 04:00, causing the large "dip" in the figure (red and blue curves).

We also used a spectrum analyzer to measure the noise at the spectrometer endstation's $\mathrm{Hg}_{1-\mathrm{x}} \mathrm{Cd}_{\mathrm{x}} \mathrm{Te}$ (MCT) infrared detector. The microspectrometer endstation was set up in the same manner as used for actual experiments on small samples, i.e. with apertures for defining the beam profile onto the specimen. Measurements of the noise with the stabilization feedback system off are shown in Figure $4 b$ (red curve) and with it on (blue curve), showing a significant improvement. 
Figure 4a.

Figure $4 b$.

\section{Conclusions}

In conclusion, we have developed and tested a beam stabilization system based on position sensitive detectors and dynamic tilt mirrors. The system has some unique features including 1) a single dichroic beamsplitter, for separating the beams for infrared (for spectroscopy) and visible light (for position sensing), situated just upstream of the instrument endstation and 2) the piezoelectric driven flexure mirror mounts in a separate upstream optical chamber. Results indicate that such a system, using mostly off-the-shelf (and LabView ${ }^{\mathrm{TM}}$ compatible) commercial components, provides useful performance benefits at a total component cost below US $\$ 12,000$. For example, a beam drift of $\sim 100$ $\mu \mathrm{m}$ or more that would occur without the stabilization system is held to an accuracy better than $1 \mu \mathrm{m}$ at the PSDs when the system is operating. By keeping the beam centered on the microspectrometer's apertures, spectrometer noise is reduced up to a factor of 10 .

\section{Acknowledgment}

This research supported by the U.S. Department of Energy under contract DE-AC0298CH10886 at Brookhaven National Laboratory. We gratefully acknowledge useful discussions with Michael Martin (ALS, LBNL) and technical contributions from Gary Nintzel (NSLS, BNL).

\section{References}

[1] Wayne R. McKinney et al, Advanced Light Source Compendium of User Abstracts (1999), LBNL 45632.

[2] D. S. Hung, Y. C. Lo, C. I. Chen and C. H. Kuo, Proc. of the 2003 Particle Accel. Conf. (2003), 3234-3236.

[3] Ph. Lerch et al, J. Synchrotron Rad. 19 (2012) 1-9.

\section{Figure Captions}

Figure 1a. Standard layout for a beam stabilization system, with tilt mirrors (TM), Position sensitive detectors (PSD) and beamsplitters (BS).

Figure 1b. Geometrical representation of the standard beam stabilization system where a PSD is placed immediately downstream of the relevant tilt mirror (TM).

Figure 2a. Alternative layout for a beam stabilization system, with tilt mirrors (TM), position sensitive detectors (PSD) and beamsplitters (BS).

Figure $2 \mathrm{~b}$. Geometrical representation of the alternative beam stabilization system where the PSDs are placed downstream of both tilt mirrors. 
Figure 2c. Geometrical representation with a lens inserted to reduce the spotsize at the PSDs. The PSDs are placed on opposite sides of the beam focus.

Figure 3 (a through e). Component layout of the beam stabilization system showing (a) electronic control modules for the PSDs and TMs, (b) schematic layout, (c) piezoelectric actuators in flexure mirror mount, (d) actual location of PSDs on endstation optical 'breadboard and (e) the infrared microspectrometer endstation instrument.

Figure 4a. Measured deviation from the two PSDs over a $\sim 5$ hour period with the stabilization feedback turned ON and OFF at approximately 15 minute intervals, demonstrating a high degree of position accuracy and quick recovery. Note the logarithmic deviation scale (abscissa).

Figure 4b. Measured noise spectrum from the spectrometer endstation's infrared detector. Noise is shown for the stabilization feedback OFF (red curve) and ON (blue curve). 

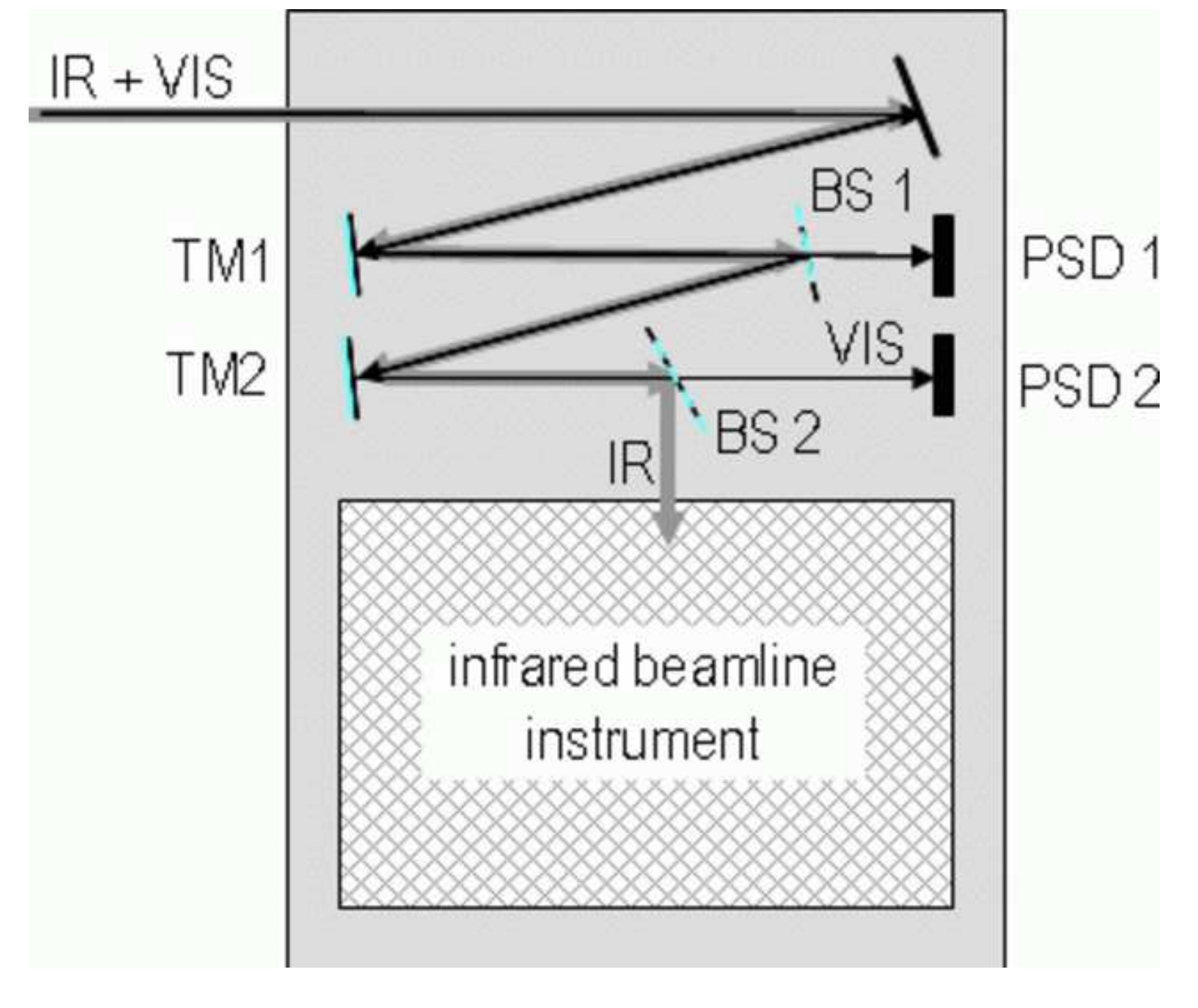


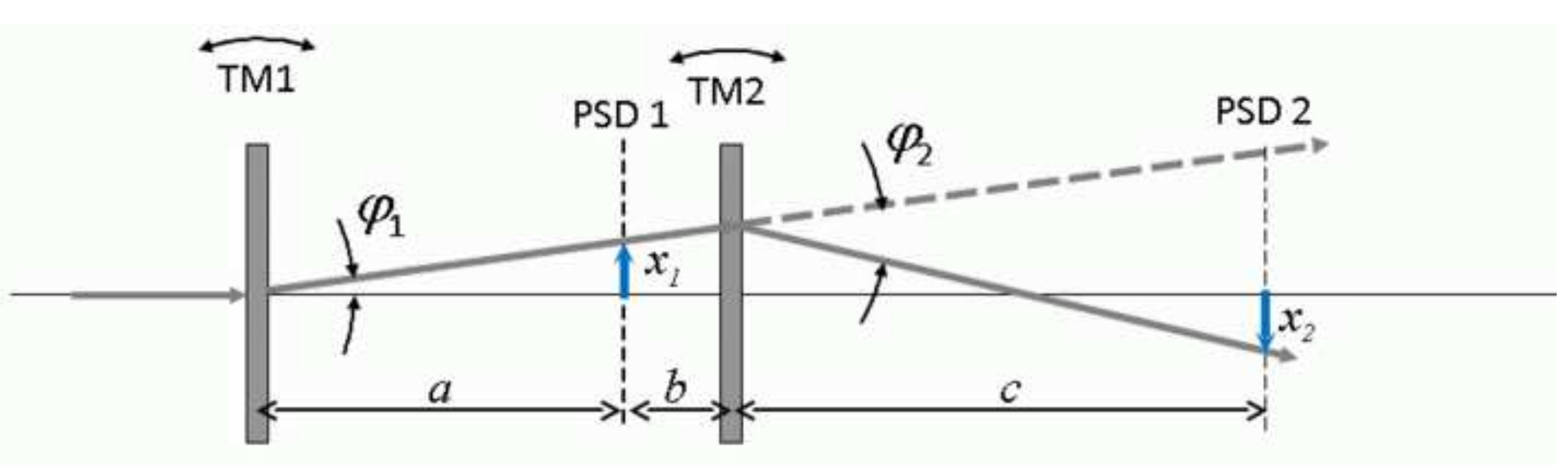




\section{TM1}

TM2
PSD 1

PSD 2

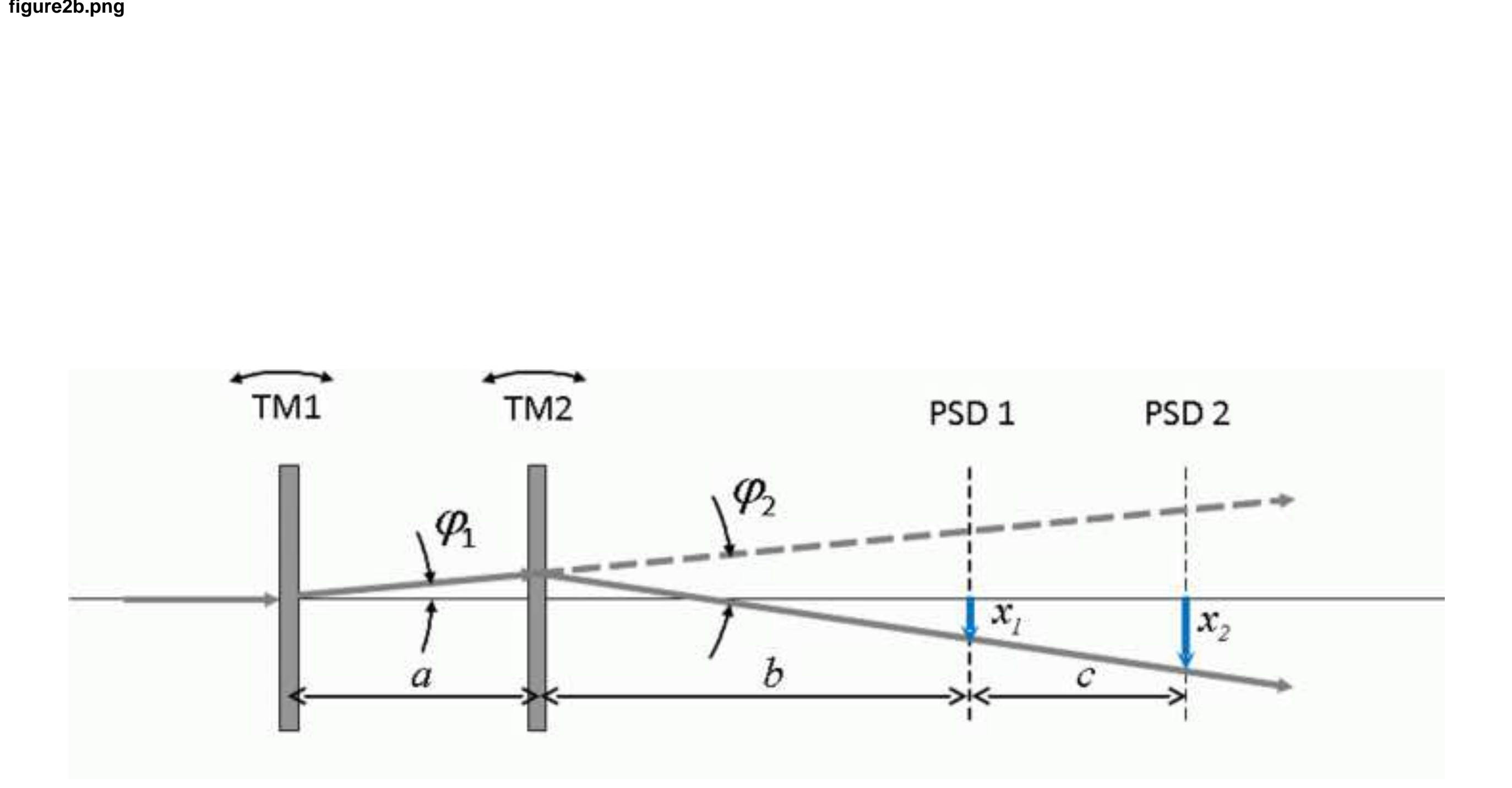

.

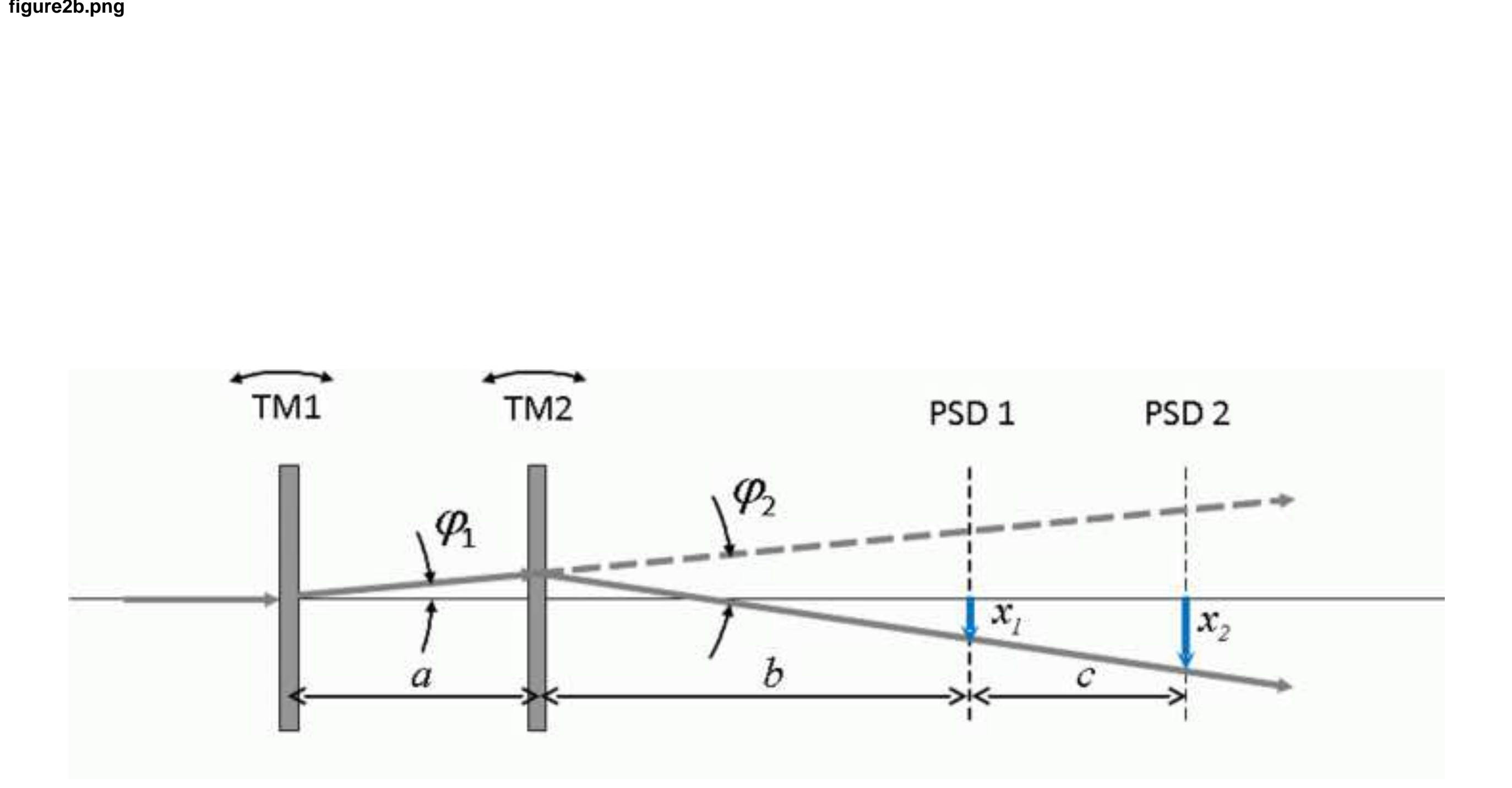




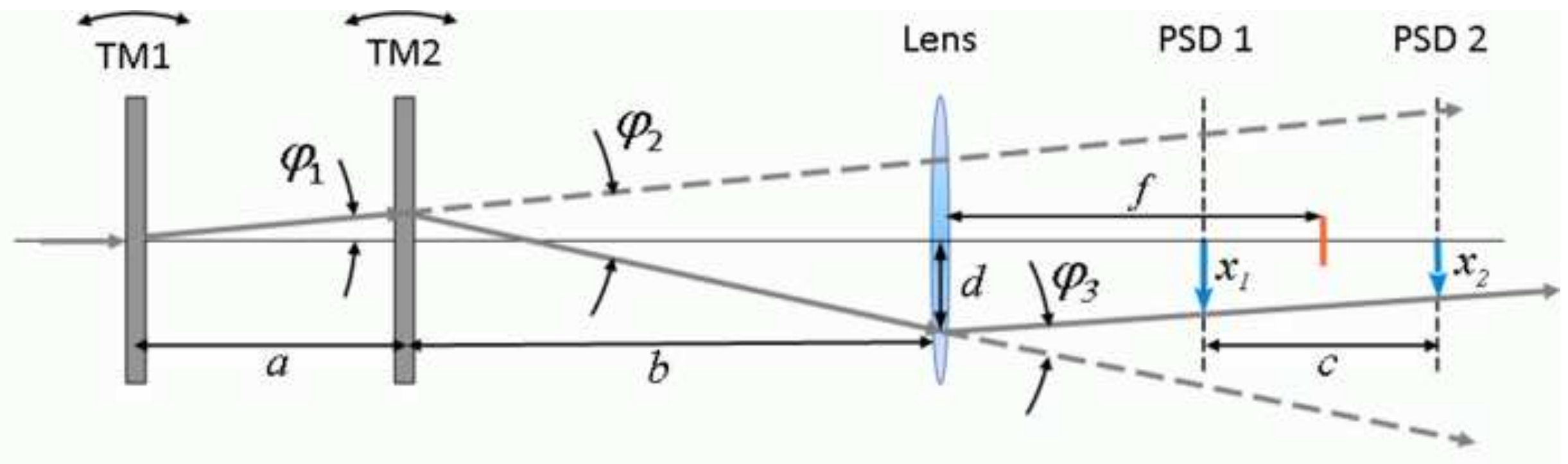


(a)

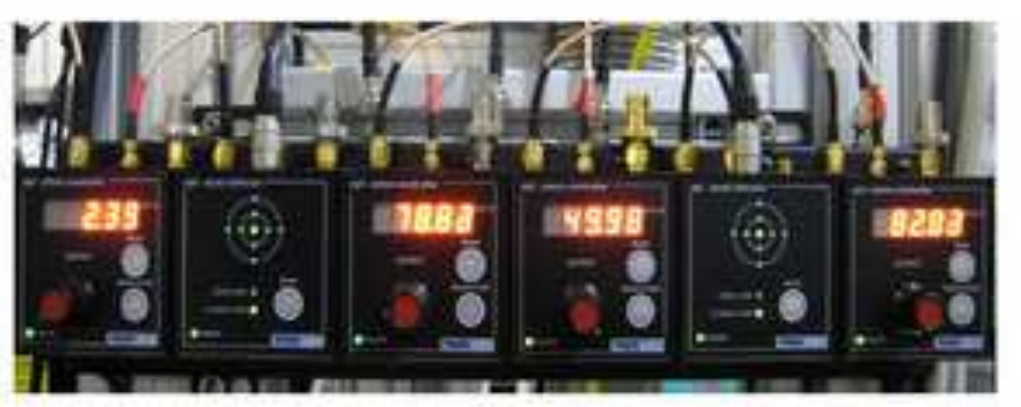

(d)

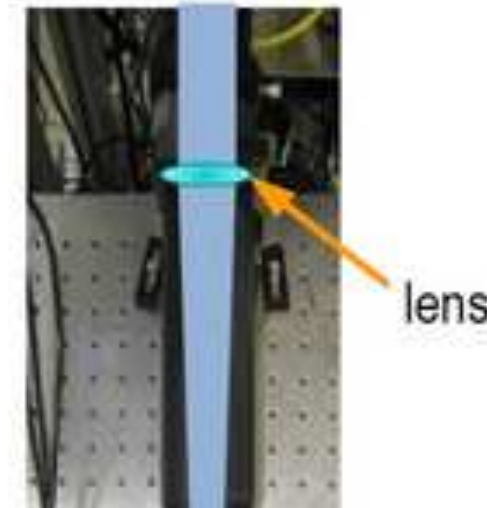

(b)

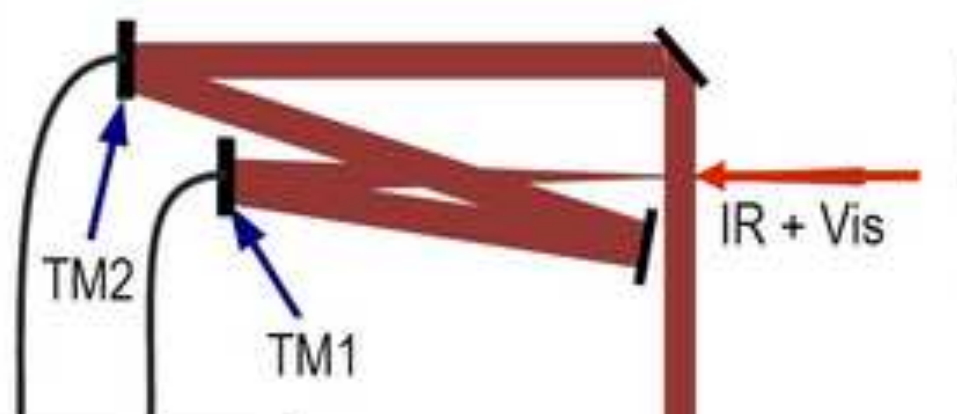

(c)

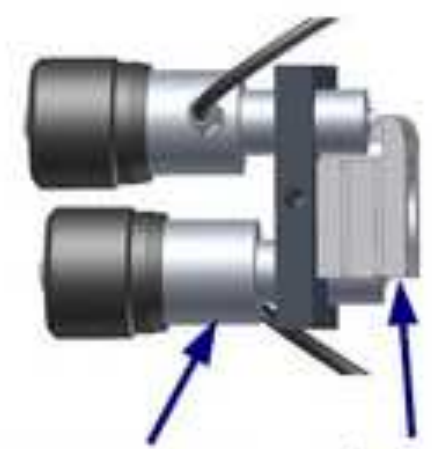

piezoelectric flexure actuator mirror mount (e)

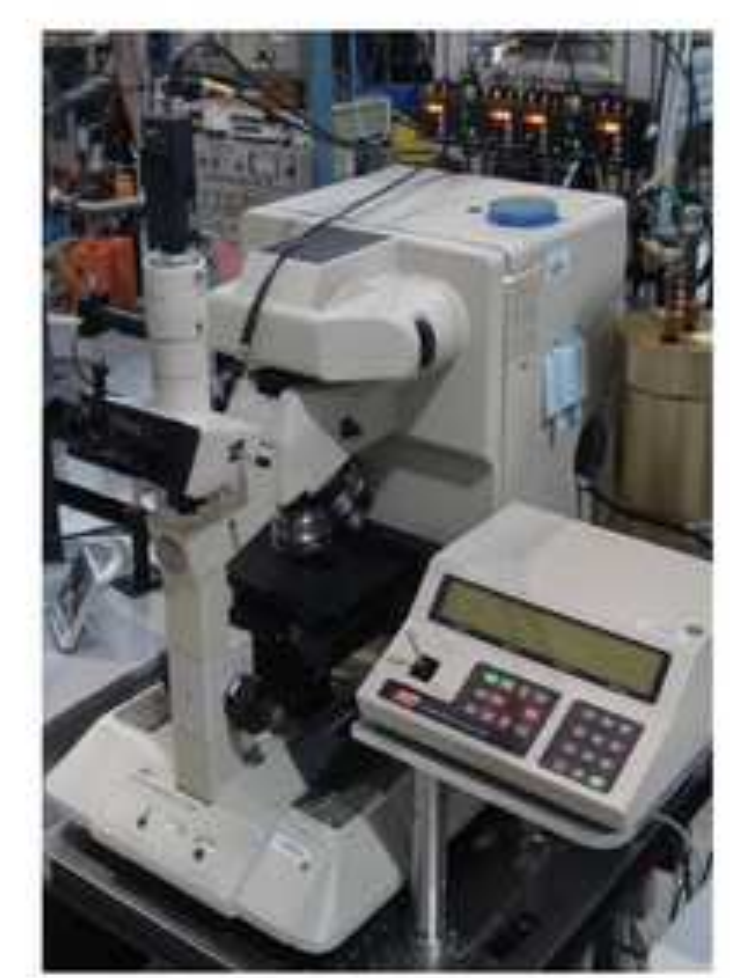

PSD 1

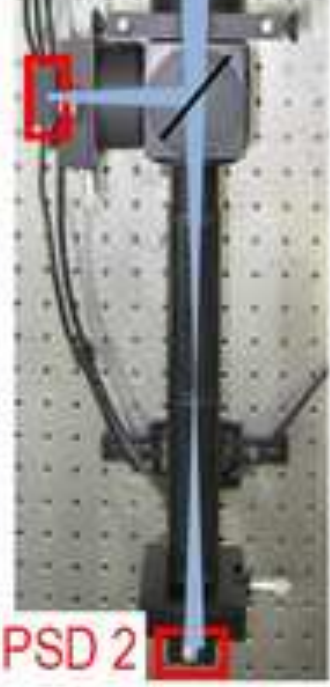

endstation table lens

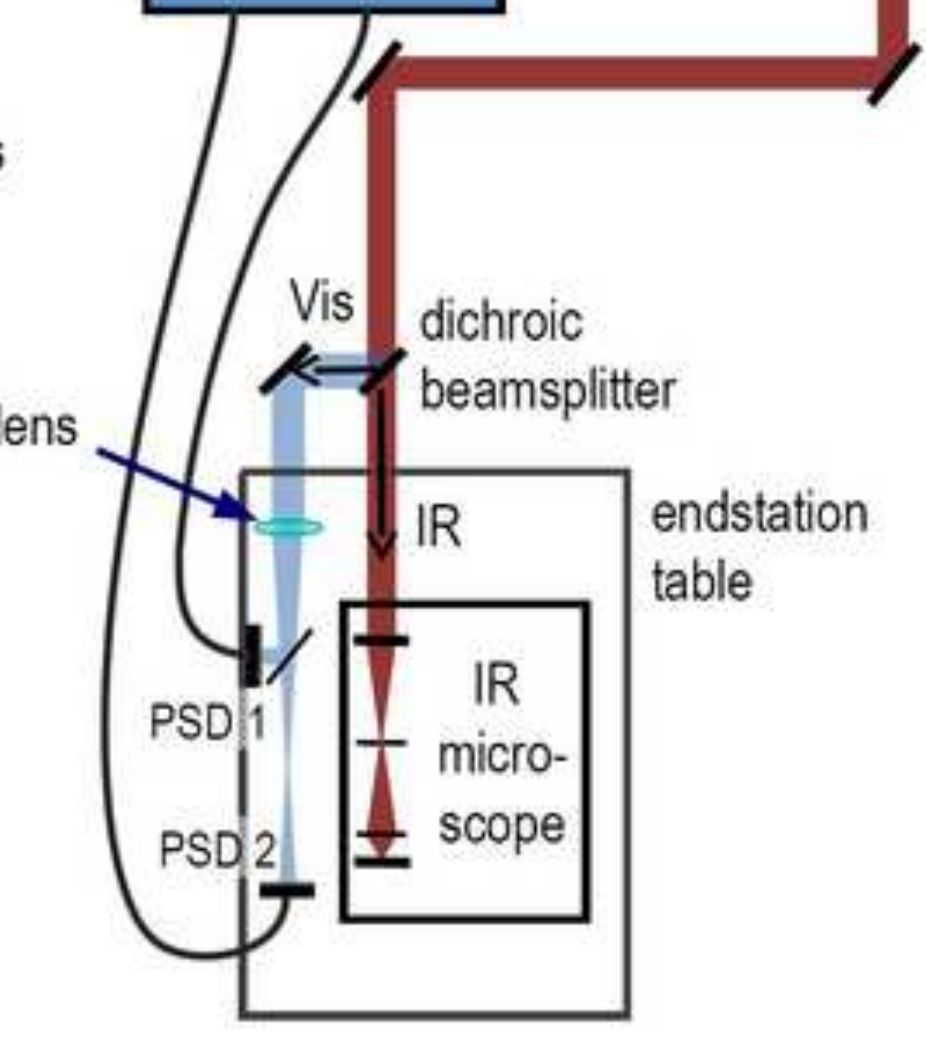

electronics 


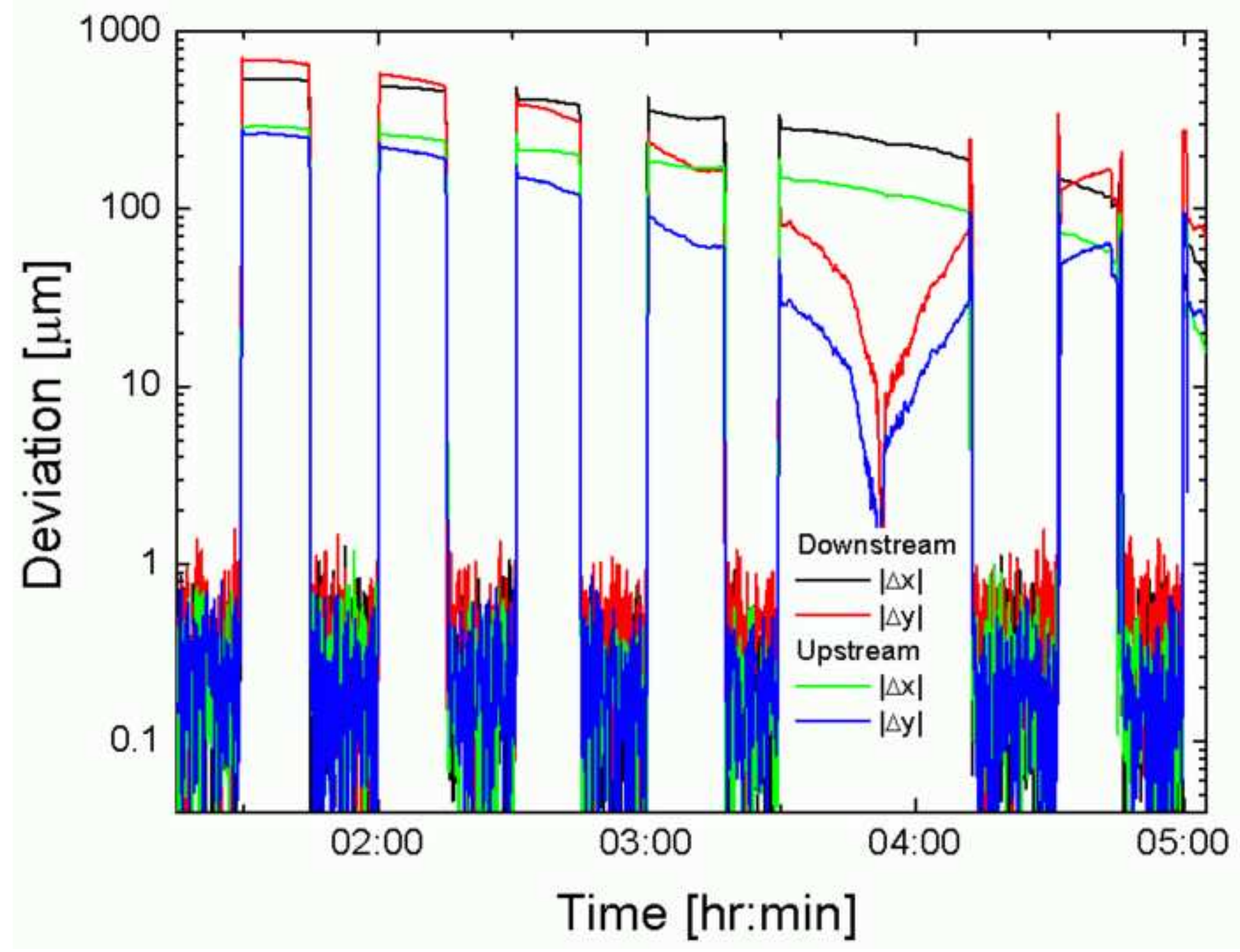




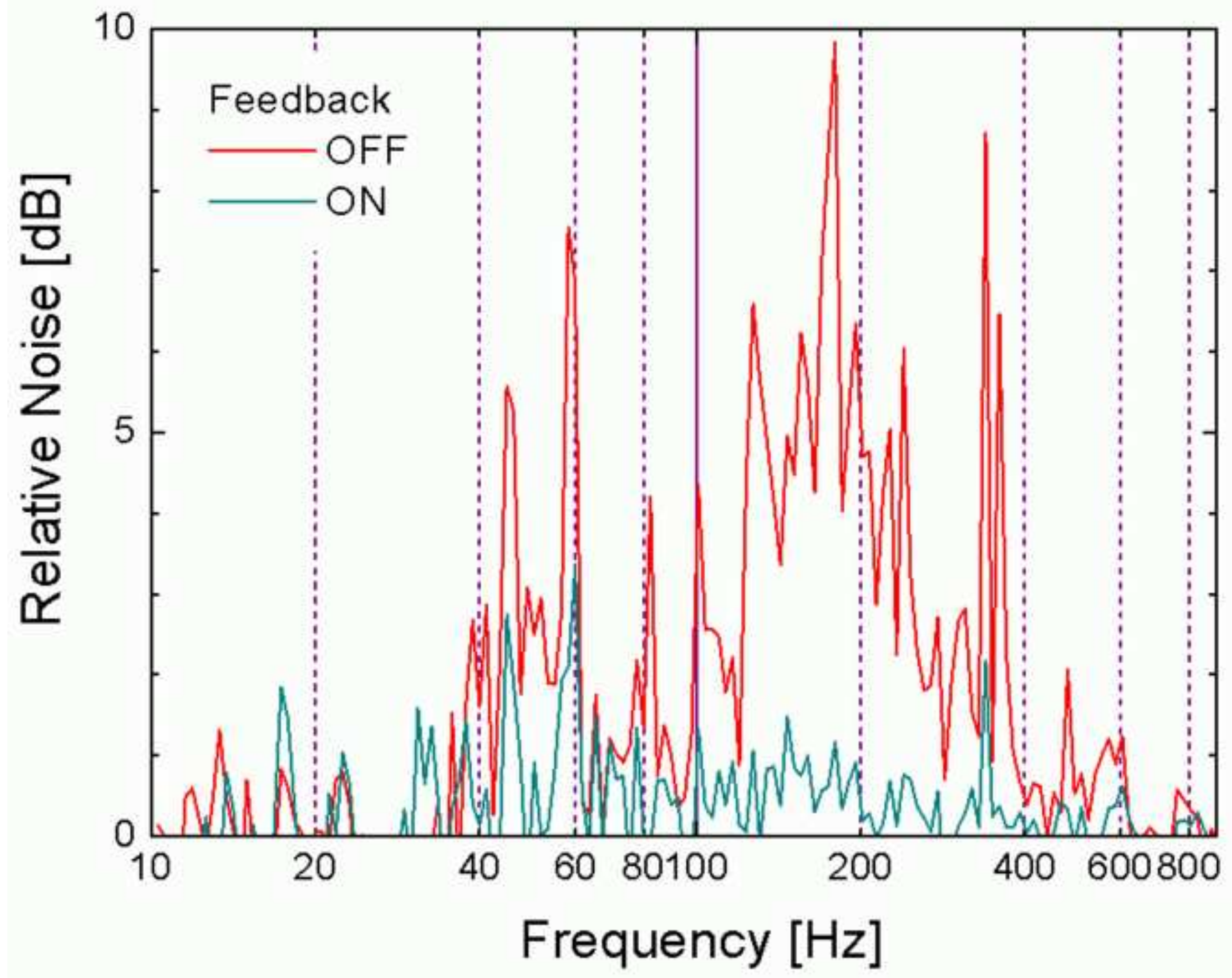



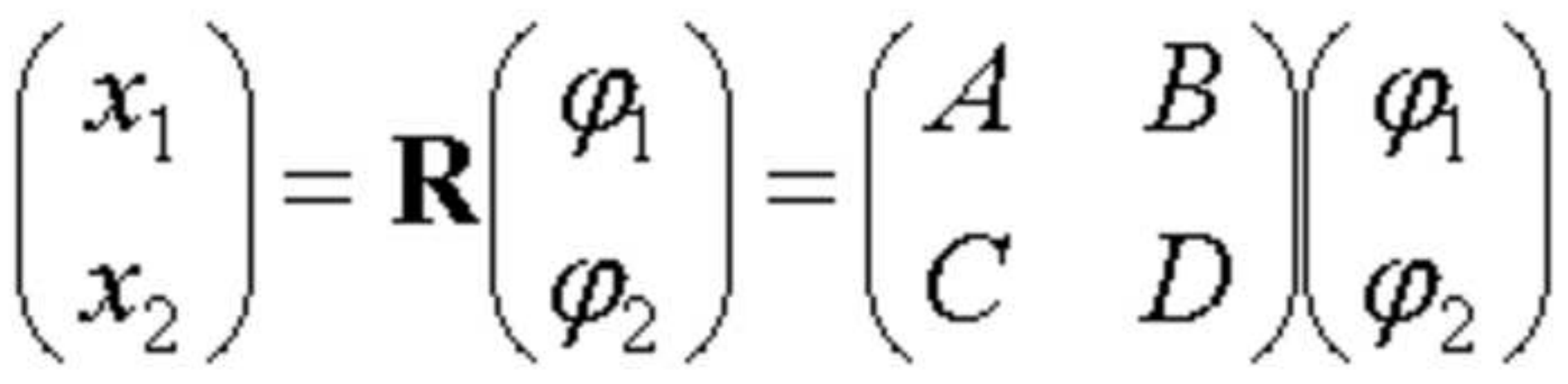


$$
K \equiv \frac{B C}{A D}=\frac{b(a+b+c)}{(a+b)(b+c)}=\frac{a b+b^{2}+b c}{a b+b^{2}+b c+a c}
$$


$x_{1}=\left[f-\frac{c}{2}+\frac{(a+b) c}{2 f}\right] \varphi_{1}+\left[f-\frac{c}{2}+\frac{b c}{2 f}\right] \varphi_{2}$

$$
x_{1}=\left[f-\frac{c}{2}+\frac{(a+b)}{2 f}\right] \varphi_{1}+\left[f-\frac{c}{2}+\frac{b c}{2 f}\right] \varphi_{2}
$$

equation $4 a$

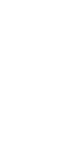

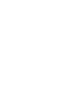$$
\text { ( }
$$

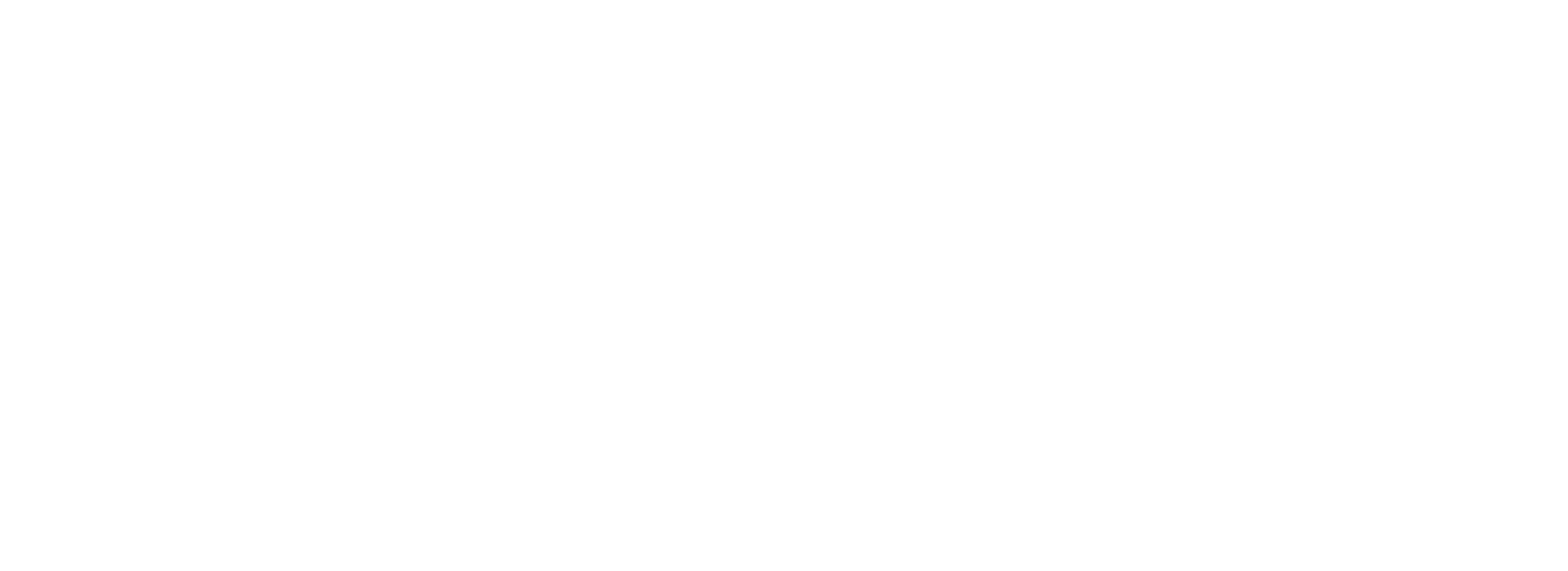


$x_{2}=\left[f+\frac{c}{2}-\frac{(a+b) c}{2 f}\right] \varphi_{1}+\left[f+\frac{c}{2}-\frac{b c}{2 f}\right] \varphi_{2}$ 